\title{
Symbolic-numeric Computation of Implicit Riquier Bases for PDE
}

\author{
Wenyuan Wu and Greg Reid \\ Dept. of Applied Mathematics, University of Western Ontario \\ London, Ontario, N6G 5B7, Canada. \\ wwu25@uwo.ca, reid@uwo.ca
}

\begin{abstract}
Riquier Bases for systems of analytic PDE are, loosely speaking, a differential analogue of Gröbner Bases for polynomial equations. They are determined in the exact case by applying a sequence of prolongations (differentiations) and eliminations to an input system of PDE.

We present a symbolic-numeric method to determine Riquier Bases in implicit form for systems which are dominated by pure derivatives in one of the independent variables and have the same number of PDE and unknowns.

The method is successful provided the prolongations with respect to the dominant independent variable have a block structure which is uncovered by Linear Programming and certain Jacobians are non-singular when evaluated at points on the zero sets defined by the functions of the PDE. For polynomially nonlinear PDE, homotopy continuation methods from Numerical Algebraic Geometry can be used to compute approximations of the points.

We give a differential algebraic interpretation of Pryce's method for ODE, which generalizes to the PDE case. A major aspect of the method's efficiency is that only prolongations with respect to a single (dominant) independent variable are made, possibly after a random change of coordinates. Potentially expensive and numerically unstable eliminations are not made. Examples are given to illustrate theoretical features of the method, including a curtain of Pendula and the control of a crane.
\end{abstract}

Categories and Subject Descriptors: G.1.8 General Terms: Algorithms, Design

Keywords: Partial Differential Equation, Riquier Bases, Linear Programming, Numerical Algebraic Geometry, Jet Spaces, Ranking, Implicit Function Theorem.

\section{INTRODUCTION}

Differential elimination algorithms apply a finite number of differentiations (prolongations) and eliminations to uncover obstructions to formal integrability. Exact differen-

Permission to make digital or hard copies of all or part of this work for personal or classroom use is granted without fee provided that copies are not made or distributed for profit or commercial advantage and that copies bear this notice and the full citation on the first page. To copy otherwise, to republish, to post on servers or to redistribute to lists, requires prior specific permission and/or a fee.

ISSAC'07, July 29-August 1, 2007, Waterloo, Ontario, Canada. Copyright 2007 ACM 978-1-59593-743-8/07/0007 ...\$5.00. tiation elimination algorithms that apply to exact polynomially nonlinear systems of PDE are given in $[2,7,14,22$, $17,16]$. Such methods enable the identification of all hidden constraints of PDE systems and the computation of initial data and associated formal power series solutions in the neighborhood of a given point. Algorithmic membership tests (specifically in the radical of a differential ideal) can be given $[2,7]$. They can ease the difficulty of numerical solution of ODE systems.

A major problem in these approaches is the exploding size of prolongations for more than one independent variable. In symbolic approaches much effort has been devoted to control the growth of this size by developing redundancy criteria (for integrability conditions), and making strong use of elimination with respect to rankings to decrease the size of the prolongations $[1,27]$. However symbolic elimination can also cause expression swell, and even in the case of one independent variable, for constrained ODE problems arising in multi-body mechanics, it is a significant problem [26].

Very little work has been done on the corresponding problems for symbolic-numeric methods. Techniques which are helpful for the symbolic case are often unstable for the approximate case, since rankings (the differential analogue of term orders) can cause pivoting on small quantities and resultant instability.

In this paper we make some progress on this problem for a certain class of PDE. For this class, only prolongations with respect to one independent variable are needed. Paradoxically rankings are important in our approach but don't cause instability since no eliminations are made. Hence we also avoid the expression swell due to the eliminations mentioned above. A suitable ranking is determined by solving an integer linear programming problem to uncover a block structure in the PDE system.

Another main idea in our paper is that such prolongations are essentially ODE like enabling us to generalize ODE techniques to the PDE case. In our case we generalize a method of Pryce for ODE in the framework of Riquier Theory. However we might imagine this being also used as a bridge for other ODE techniques (e.g. that of Sedoglavic [21]).

In particular, we give methods for computing approximate implicit Riquier Bases for square systems of analytic PDE.

There already exist exact methods for computing Riquier Bases for non-square polynomially nonlinear PDE together with an input ranking of derivatives [18]. However these exact methods may not succeed if the intermediate systems, can not be solved explicitly for their leading derivatives.

For polynomially nonlinear PDE, our approximate Riquier 
Basis method uses an approximate method, homotopy continuation, to by-pass this difficulty. From a given set of solutions of a system of similar structure, homotopy paths converge to points on the zero set of the functions in the prolongations of the PDE system. It is these points that are used to verify the conditions of the Implicit Function Theorem, allowing the implicit solution of the given functions for their leading derivatives. For background on the homotopy methods, constituting the new area of Numerical Algebraic Geometry, please see the book [24].

In addition our method yields the method of Pryce [13] for systems of differential algebraic equations as a special case. Prolongation will usually introduce more equations as well as more (jet) variables, but this is not always true. If some equations after differentiation do not introduce new variables for whole system, then there is the possibility that the dimension of the system is lowered, since generically the system's dimension is the number of its variables minus the number of its equations. Pryce [13] proposed a method to detect such "chances" that minimize the dimension by taking advantage of the special structure of some systems. Pryce's method was the generalization of a method developed by Pantiledes. Ilie et al [6] show Pryce's method, can be extended to give a polynomial cost method for numerical solution of differential algebraic equations.

\section{ZERO SET OF PDE}

Let $\mathbb{F}$ be a field ( $\mathbb{R}$ or $\mathbb{C}$ in this paper), $x=\left(x_{1}, \cdots, x_{n}\right)$ be the independent variables and $u=\left(u^{1}, \cdots, u^{m}\right)$ be the dependent variables for a system of PDE. The usual commutative approaches to differential algebra and differential elimination theory $[18,2]$ consider a set of indeterminates $\Omega=\left\{v_{\alpha}^{i} \mid \alpha=\left(\alpha_{1}, \cdots, \alpha_{n}\right) \in \mathbb{N}^{n}, i=1, \cdots, m\right\}$ where each member of $\Omega$ corresponds to a partial derivative by:

$v_{\alpha}^{i} \leftrightarrow\left(\mathbf{D}_{x_{n}}\right)^{\alpha_{n}} \cdots\left(\mathbf{D}_{x_{1}}\right)^{\alpha_{1}} u^{i}\left(x_{1}, \cdots, x_{n}\right):=\mathbf{D}^{\alpha} u^{i}\left(x_{1}, \cdots, x_{n}\right)$.

Formal commutative total derivative operators are introduced to act on members of $\Omega$ by a unit increment of the $i$-th index of their vector subscript: $\mathbf{D}_{x_{i}} v_{\alpha}^{k}:=v_{\alpha+1_{i}}^{k}$ where $\alpha+1_{i}=\left(\alpha_{1}, \ldots, \alpha_{i}+1, \ldots, \alpha_{n}\right)$. The usual total derivatives $\mathbf{D}_{x_{i}}$ act on functions of $\{x\} \cup \Omega$ by:

$$
\mathbf{D}_{x_{i}}=\frac{\partial}{\partial x_{i}}+\sum_{v \in \Omega}\left(\mathbf{D}_{x_{i}} v\right) \frac{\partial}{\partial v}
$$

where $\frac{\partial}{\partial v}$ are the usual partial derivatives.

A $q$-th order differential system with $\ell$ equations is associated with a locus (or zero set) of points

$$
Z(f):=\left\{\left(x, v_{\alpha}^{i}\right) \in J^{q}\left(\mathbb{F}^{n}, \mathbb{F}^{m}\right): f^{k}\left(x, v_{\alpha}^{i}\right)=0, k=1, \ldots, \ell\right\}
$$

where $J^{q}\left(\mathbb{F}^{n}, \mathbb{F}^{m}\right) \simeq \mathbb{F}^{n} \times \mathbb{F}^{m} \times \mathbb{F}^{m_{1}} \times \ldots \times \mathbb{F}^{m_{q}}$ is the jet space of order $q$ and $f^{k}: J^{q}\left(\mathbb{F}^{n}, \mathbb{F}^{m}\right) \rightarrow \mathbb{F}, k=1, \ldots, \ell$ are the maps defining the differential equations. Here $m_{r}:=m$. $\left(\begin{array}{c}r+n-1 \\ r\end{array}\right)$ is the number of jet variables corresponding to $r$-th order derivatives.

One class of systems considered in this paper will be differential polynomials in $\mathbb{F}\left[x_{1}, \ldots, x_{n} ; v_{\alpha}^{i}:|\alpha| \geq 0\right]$, the ring of all polynomials over $\mathbb{F}$ in the infinite set of indeterminates $\{x\} \cup \Omega$, where $|\alpha|=\alpha_{1}+\cdots \alpha_{n}$. The other case is that where the $f^{k}$ are $\mathbb{F}$-analytic functions in a neighborhood of a point $\left(x^{0},\left(v_{\alpha}^{i}\right)^{0}\right)$ which by our finiteness restriction can be taken in $J^{q}$. We restrict to $f^{k}$ being functions of finitely many indeterminates. We alert the reader that although we occasionally use Jet notation, we always work locally over some $\mathbb{F}$-Euclidian space. So we don't use the more global geometric features of Jet Geometry, such as bundles, contact structures, etc (see [22]).

The pendulum is a simple example of differential equations (commonly called differential algebraic equations or DAE) that arise frequently in applications. As a matter of terminology, throughout this paper we will use the term ODE to include DAE. Such systems are ubiquitous in multi-body dynamics. From CAD like graphical descriptions of links, joints, motors, etc, there are several software packages (e.g. Adams, Dads and WorkingModel [23]), that automatically produce the equations of motion, using Lagrangian mechanics formulations.

EXAMPLE 2.1. [The Pendulum] For the pendulum of unit mass, under constant gravity, we have

$$
\begin{aligned}
X_{t t}+\lambda X & =0, \\
Y_{t t}+\lambda Y & =-g, \\
X^{2}+Y^{2} & =1 .
\end{aligned}
$$

Here

$$
\begin{gathered}
Z(f)=\left\{\left(t, X, Y, \lambda, X_{t}, Y_{t}, \lambda_{t}, X_{t t}, Y_{t t}, \lambda_{t t}\right) \in J^{2}:\right. \\
\left.X_{t t}+\lambda X=0, Y_{t t}+\lambda Y+g=0, X^{2}+Y^{2}-1=0\right\}
\end{gathered}
$$

is a 7 dimensional submanifold of $\mathbb{F}^{10} \simeq J^{2}$.

\section{RANKINGS OF DERIVATIVES}

A detailed formal treatment of this subject, and the classification of all such rankings are given in Rust et al. [18]. Rankings are fundamental in Differential Algebra [8].

Definition 3.1 (RAnking [18]). A positive ranking $\prec$ of $\Omega$ is a total ordering on $\Omega$ which satisfies:

$$
\begin{aligned}
v_{\alpha}^{i} \prec v_{\beta}^{j} & \Rightarrow v_{\alpha+\gamma}^{i} \prec v_{\beta+\gamma}^{j}, \\
v_{\alpha}^{i} & \prec v_{\alpha+\gamma}^{i},
\end{aligned}
$$

for all $\alpha, \beta, \gamma \in \mathbb{N}^{n}$.

Let $\operatorname{HD} f$ denote the greatest member in $\Omega$ in $f$ with respect the ranking $\prec$.

EXAMPLE 3.1. An example of a ranking for the Pendulum system given in Example 2.1 is:

$$
X \prec Y \prec \lambda \prec X_{t} \prec Y_{t} \prec \lambda_{t} \prec X_{t t} \prec Y_{t t} \prec \lambda_{t t} \prec \cdots
$$

It is easily seen that (6) is invariant under differentiation, so (4) is satisfied. In addition any derivative of a member is greater than itself, so (5) is satisfied. In this ranking $\operatorname{HD}\left(X_{t t}+\lambda X\right)=X_{t t}, \operatorname{HD}\left(Y_{t t}+\lambda Y-g\right)=Y_{t t}$, and $\operatorname{HD}\left(X^{2}+\right.$ $\left.Y^{2}-1\right)=Y$.

There are many ways to specify a ranking. In this paper we use a matrix representation following Riquier and Rust $[18,19]$. First we introduce a map $\psi$ from $\Omega$ to $\mathbb{Z}^{m+n}$ :

$$
\psi: \frac{\partial^{\alpha_{1}+\cdots+\alpha_{n}} u^{j}}{\partial x_{1}^{\alpha_{1}} \cdots \partial x_{n}^{\alpha_{n}}} \mapsto\left(0, \cdots, 0,1,0, \cdots, 0, \alpha_{1}, \cdots, \alpha_{n}\right)^{t}
$$

where the " 1 " appears in $j$ th coordinate.

An ordering of the elements in $\mathbb{Z}^{m+n}$ denoted by $<$ is defined by lexical order (comparing the values at the first coordinate, then the second coordinate, and so on). 


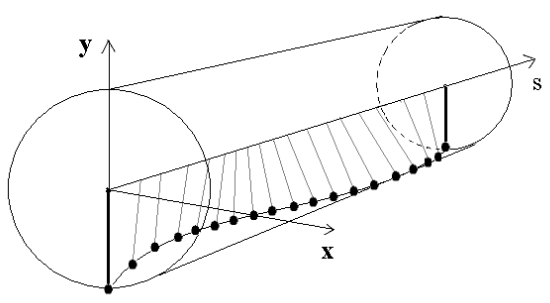

Figure 1: Pendulum Curtain

Definition 3.2. [Ranking by Matrix] Suppose $M$ is an $l \times(m+n)$ matrix with nonnegative integer entries and satisfies: $\theta \neq \tau \Rightarrow M \cdot \psi(\theta) \neq M \cdot \psi(\tau)$. We define $\prec_{M}$ to be a ranking with respect to $M$, if $\theta, \tau \in \Omega$, we have $\theta \prec_{M} \tau \Leftrightarrow$ $M \cdot \psi(\theta)<M \cdot \psi(\tau)$. Here $M$ called a matrix representation of this ranking. And $\theta \preceq_{M} \tau$ means $\theta \prec_{M} \tau$ or $\theta=\tau$.

\section{SIGNATURE MATRIX OF T-DOMINATED SYSTEMS USING RANKINGS}

The methods developed in this paper are applicable to a class of PDE that are dominated by pure derivatives in one of their independent variables.

Examples of such PDE include those of Cauchy-Kovaleskya type such as hyperbolic equations (e.g. the wave equation $\left.u_{t t}=c^{2} u_{x x}\right)$. Equations of parabolic type, such as the classical Heat equation $u_{t}=u_{x x}$ are also included. In these cases the dominating variable, is the time $t$. PDE of elliptic type are included in this class, such as the Cauchy-Riemann equations: $\left\{u_{x}=v_{y}, v_{x}=-u_{y}\right\}$.

Our main illustrative example is:

EXAMPLE 4.1. [Pendulum Curtain] Consider a curtain made of many pendula hanging under gravity $g$ as shown in Figure 1. The Pendula are restricted to move on the surface of the cylinder and in planes perpendicular to the $s$ axis displayed in Figure 1. The pendula form a continuous curtain in the limit. For small deviations from the vertical equilibrium position the equations for $X(t, s), Y(t, s)$ and Lagrange multiplier $\lambda(t, s)$ for the continuous curtain are:

$$
\begin{aligned}
X_{t t}+\lambda X & =\kappa X_{s s} \\
Y_{t t}+\lambda Y+g & =\kappa Y_{s s} \\
\frac{1}{2}\left(X^{2}+Y^{2}-1\right) & =0
\end{aligned}
$$

Note that when $\kappa=0$ this reduces to the simple pendulum equations given in Example 2.1.

By a pure derivative with respect to an independent variable $x_{i}$, we mean a derivative of form $\left(\frac{\partial}{\partial x_{i}}\right)^{k} u^{j}$ where $k \in$ $\mathbb{N}$. By Definition 4.1 given later, a PDE system which is dominated by pure derivatives with respect to an independent variable $x_{i}$, must at least contain such a derivative in each of its equations. The Pendulum Curtain system (8) satisfies this requirement with respect to $t$. A physically important class of PDE which are dominated by pure derivatives in time consists of evolution PDE. In that case the time derivatives can be expressed as functions of spatial derivatives.
For two independent variables $t, x$ and for each $u^{j}$, a ranking consistent with such systems should satisfy:

$$
u^{j} \prec u_{x}^{j} \prec u_{x x}^{j} \prec \cdots \prec u_{t}^{j} \prec u_{t x}^{j} \prec \cdots
$$

It is easy to extend this (partial) ranking to the case when $x$ is a vector (e.g. using lexical order on $x$ ).

For the pendulum curtain example, the differential order of $t$ is more important than spatial derivatives. More generally, we can focus on a special independent variable $x_{k}$ and for notational convenience denote $x_{k}$ by $t$. However we warn the reader that $t$ may not represent time for some physical $t$-dominated systems. For example the elliptic CauchyRiemann equations $\left(u_{x}=v_{y}, v_{x}=-u_{y}\right)$ are $x$-dominated.

We hide the details about the differential order of the other independent variables by defining a weight map $\varphi$ : $\Omega \rightarrow \mathbb{R}$ as follows:

$$
\varphi\left(v_{\alpha}^{i}\right):= \begin{cases}\alpha_{k}, & \text { if } \alpha_{p}=0, \text { for any } \mathrm{p} \neq \mathrm{k} ; \\ \alpha_{k}+\epsilon, & \text { otherwise. }\end{cases}
$$

The leading derivative of each equation $R_{i}$ with respect to each $u^{j}$ using the ranking (9), is denoted by $\operatorname{LD}\left(R_{i}, u^{j}\right)$. Applying (10) to the leading derivatives of $R$, we obtain an $\ell \times m$ matrix $\left(\sigma_{i, j}\right)$ which is called the signature matrix of $R$ (see Pryce [13] for the ODE case):

$$
\left(\sigma_{i, j}\right)(R):= \begin{cases}\varphi\left(\operatorname{LD}\left(R_{i}, u^{j}\right)\right), & \text { if } R_{i} \text { depends on } u^{j} \\ -\infty, & \text { otherwise }\end{cases}
$$

It is easy to show that $\left(\sigma_{i, j}\right)\left(\mathbf{D}_{t} R\right)=\left(\sigma_{i, j}\right)(R)+1^{\ell \times m}$, where $1^{\ell \times m}$ is a matrix with all entries equal to 1 .

We define the leading class of derivatives by

$$
\operatorname{LCD}(R):=\left\{\operatorname{LD}\left(R, u^{j}\right): 1 \leq j \leq m\right\} .
$$

These are the highest derivatives of $u^{j}$ appearing in $R$.

If for each equation of $R$, the leading class of derivatives are pure $t$-derivatives, then regarding the other independent variables as parameters the PDE has an ODE-like structure. Then we can consider the other independent variables as parameters to regard the PDE as ODE. To study the PDE with this structure, we introduce a new concept:

Definition 4.1. [t-Dominated System] We say $R$ is dominated by pure derivatives in the independent variable $t$ if there is no $\epsilon$ appearing in $\left(\sigma_{i, j}\right)(R)$. For notational simplicity, we also call $R$ at-dominated system.

Such $t$-dominated systems are not as special as they appear.

Proposition 4.2. [Genericity of t-dominated Systems] A generic $\mathbb{F}$-analytic or polynomially nonlinear PDE system $R$ with order $k$ is $t$-dominated. Any $\mathbb{F}$-analytic or polynomially nonlinear PDE system $R$ with order $k$ is $t$-dominated after a random linear coordinates transformation in the independent variables with coefficients in $\mathbb{F}$.

Proof. Let $R$ be a generic PDE. So each $R_{i}$ contains all pure $t$ derivatives with order $k$, which are the leading class of derivatives with respect to Ranking (9). For any nonlinear PDE $R$, after a random linear coordinate change, any derivative with order $k$ becomes a linear combination of all the $k$ th order derivatives. So $R$ contains all pure $k$ th order $t$ derivatives which are the leading class of derivatives.

REMARK 4.3. A symbolic random linear coordinates transformation often destroys the sparsity of the original system, 
which causes a dramatic increase in size of the system if subsequent eliminations are applied. However our numeric transformation in fixed precision lessens expression growth. Also, as we will show, no eliminations are involved in our method.

\section{GENERALIZING PRYCE'S PROLONGATION METHOD TO PDE}

Let $R$ be a square (i.e. \#equations $=$ \#unknowns) and $t$-dominated system. From Section 4 , the signature matrix $\left(\sigma_{i, j}\right)(R)$ contains information on differential order and ignores details on the degrees and coefficients of a system $R$. We introduce a fast method based on $\left(\sigma_{i, j}\right)(R)$ to differentiate (prolong) $R$ with respect to $t$. Pryce's prolongation method for square ODE is a special case. It yields a local existence and uniqueness result (equivalently all local constraints in initial conditions for $R$ are determined). We obtain a local existence and uniqueness result for square PDE which is given in Section 6.

If we consider $R$ as ODE (the only independent variable is $t$ ). Pryce's method [13] finds all the local constraints for a large class of square ODE using only prolongation. We generalize this construction to PDE. To be precise, the description of this construction is given in this section, but the detailed justification of its properties under certain conditions is given in Section 6. Suppose $R_{i}$ is differentiated $c_{i}$ times $\left(c_{i} \geq 0\right)$. The new system after differentiation is denoted by $\overline{\mathbf{D}}_{t}^{c} R$. Suppose the highest order of $u^{j}$ appear in $\mathbf{D}_{t}^{c} R$ is $d_{j}$. From the definition of $\left(\sigma_{i, j}\right)$, clearly $d_{j}$ is the largest of $c_{i}+\sigma_{i j}$, which implies that

$$
d_{j}-c_{i} \geq \sigma_{i j}, \text { for all } i, j
$$

Obviously there are at most $\sum d_{j}+m$ pure $t$-derivative jet variables and $\sum c_{i}+m$ equations in $\mathbf{D}_{t}^{c} R$ (considering independent variables and all non- $t$-derivatives as parameters). We can embed $\mathbf{D}_{t}^{c} R$ into a $\sum d_{j}+m$ dimensional space. If each equation drops the dimension of the zero set of $\mathbf{D}_{t}^{c} R$ by one, then the dimension of $\mathbf{D}_{t}^{c} R$ is $\sum d_{j}-\sum c_{i}$. Roughly speaking, to find all the constraints is equivalent to minimizing the dimension of $\mathbf{D}_{t}^{c} R$. This can be formulated as an integer linear programming problem in the variables $c=\left(c_{1}, \ldots, c_{m}\right)$ and $d=\left(d_{1}, \ldots, d_{m}\right)$ :

$$
\left\{\begin{aligned}
\text { Minimize } & z=\sum d_{j}-\sum c_{i} \\
\text { where } & d_{j}-c_{i} \geq \sigma_{i j} \\
& c_{i} \geq 0
\end{aligned}\right.
$$

The computation of $c$ and $d$ which only involves the information on differential order and is consequently very fast.

REMARK 5.1. However this linear programming problem may not have solution. If we consider its dual problem in the sense of linear programming, which is an Assignment Problem. The task is to choose just one element in each row and column of the signature matrix, then maximize the sum of these $m$ elements. The maximum is called the Maximal Transversal Value. If this value exists, then (13) has finite solution. Like Pryce's method [13], we always assume that the maximal transversal value exists in this paper.

EXAMPLE 5.1. Note that Example (4.1) is t-dominated (and also s-dominated). Thus we can apply the method above for PDE. The signature matrix for the above system with

\begin{tabular}{|c|c|c|c|c|}
\hline$B_{0}$ & $B_{1}$ & $\cdots$ & $B_{k_{c}-1}$ & $B_{k_{c}}$ \\
\hline$R_{1}^{(0)}$ & $R_{1}^{(1)}$ & $\cdots$ & $R_{1}^{\left(c_{1}-1\right)}$ & $R_{1}^{\left(c_{1}\right)}$ \\
& $R_{2}^{(0)}$ & $\cdots$ & $R_{2}^{\left(c_{2}-1\right)}$ & $R_{2}^{\left(c_{2}\right)}$ \\
& & $\vdots$ & $\vdots$ & $\vdots$ \\
& & $R_{m}^{(0)}$ & $\cdots$ & $R_{m}^{\left(c_{m}\right)}$ \\
\hline
\end{tabular}

Table 1: The triangular block structure of $\mathbf{D}_{t}^{c} R$. For $0 \leq i<k_{c}, B_{i}$ has fewer jet variables than $B_{i+1}$.

columns corresponding to $X, Y$ and $\lambda$ from left to right is:

$$
\left(\sigma_{i, j}\right)=\left(\begin{array}{ccc}
2 & -\infty & 0 \\
-\infty & 2 & 0 \\
0 & 0 & -\infty
\end{array}\right)
$$

Recall that $c_{i}$ means the $i$-th equation needs to be differentiated $c_{i}$ times $\left(c_{i} \geq 0\right)$ and $d_{j}$ is the highest order of $u^{j}$ after the prolongation. Then (13) is

$$
\left\{\begin{aligned}
\text { Minimize } & z=d_{1}+d_{2}+d_{3}-c_{1}-c_{2}-c_{3}, \\
\text { where } & d_{1}-c_{1} \geq 2, \quad d_{1}-c_{2} \geq-\infty, \quad d_{1}-c_{3} \geq 0 \\
& d_{2}-c_{1} \geq-\infty, \quad d_{2}-c_{2} \geq 2, \quad d_{2}-c_{3} \geq 0 \\
& d_{3}-c_{1} \geq 0, \quad d_{3}-c_{2} \geq 0, \quad d_{3}-c_{3} \geq-\infty \\
& c_{1} \geq 0, \quad c_{2} \geq 0, \quad c_{3} \geq 0
\end{aligned}\right.
$$

Solving this integer linear programming problem by LPSolve in the Optimization package of Maple10, we obtain

$$
\begin{aligned}
& c_{1}=0, \quad c_{2}=0, \quad c_{3}=2 \\
& d_{1}=2, \quad d_{2}=2, \quad d_{3}=0
\end{aligned}
$$

After we obtain the number of prolongation steps $c_{i}$ for each equation, we can construct the partial prolonged system $\mathbf{D}_{t}^{c} R$ using $c$.

We also point out that $\mathbf{D}_{t}^{c} R$ has a favorable block triangular structure which enables us to compute points on $Z\left(\mathbf{D}_{t}^{c} R\right)$ more efficiently. Without loss of generality, we assume $c_{1} \geq c_{2} \geq \cdots \geq c_{m}$, and let $k_{c}=c_{1}$, which is closely related to the index of system $R$ (see [13] for more details about the index). Then we can partition $\mathbf{D}_{t}^{c} R$ into $k_{c}+1$ parts (see Table 5).

For each $B_{i}, 0 \leq i \leq k_{c}$, we denote the leading class of variables by $U_{i}:=\operatorname{LCD}\left(B_{i}\right)$ and define the Jacobian Matrix

$$
\mathcal{J}_{i}:=\left(\frac{\partial B_{i}}{\partial U_{i}}\right) \text {. }
$$

Proposition 5.2. Let $\mathcal{J}\left(\boldsymbol{D}_{t}^{c} R\right):=\left\{\mathcal{J}_{i}\right\}$ be the set of Jacobian matrices of $\left\{B_{i}\right\}$. For any $0 \leq i<j \leq k_{c}, \mathcal{J}_{i}$ is a sub-matrix of $\mathcal{J}_{j}$. Moreover, if $\mathcal{J}_{k_{c}}$ has full rank, then any $\mathcal{J}_{i}$ also has full rank.

Proof. The first result is by the chain rule and the fact that if $\theta$ is the leading variable of a $\operatorname{PDE} F$ then $\theta_{t}$ is the leading variable of $\mathbf{D}_{t} F$.

Because $\mathcal{J}_{k_{c}}$ is an $m \times m$ full rank, each row is linearly independent. Since $\mathcal{J}_{i}$ is a sub-matrix of $\mathcal{J}_{k_{c}}$, we can assume it consists of the first $p$ rows and first $q$ columns of $\mathcal{J}_{k_{c}}$, where $q$ is the number of elements in $U_{i}$. If $q=m$, then $\operatorname{rank}\left(\mathcal{J}_{i}\right)=p$. If $q<m$, then the entries in first $p$ rows and last $m-q$ columns must be 0 . So $\operatorname{rank}\left(\mathcal{J}_{i}\right)=p$.

In the following section we will show that the output of the $t$-prolongation implicitly yields a Riquier Basis for which an associated existence theorem is available. 


\section{THE FORMAL RIQUIER EXISTENCE THEOREM}

In this section, we state Theorem 6.5 for the existence and uniqueness of formal power series solutions of a Riquier Basis. This theorem is the result of a Gröbner style development and extension of Riquier's classical existence results for PDE. The details can be found in the works of Rust et al. $[19,18]$. The corresponding exact symbolic differential elimination algorithms were implemented [27] in distributed Maple; which also refers to applications of the algorithms.

Given a ranking of partial derivatives, such bases are in solved form with respect to leading derivatives. They are symbolically determined by successively including integrability conditions and performing eliminations on the resulting systems. The solved form requirement means that in the exact case they are essentially restricted to PDE which are linear in their highest derivatives. Closely related to Riquier Bases are Schwarz's Janet Bases [20].

We say that $f$ is $\prec$-monic with respect to a ranking $\prec$ if $f$ has the form $f=\operatorname{HD} f+g$, with $\operatorname{HD} g \prec \operatorname{HD} f$. For example the equation $X^{2}+Y^{2}-1=0$ of the Pendulum system of (3) is not $\prec$-monic with respect to the ranking given in (6) since it is nonlinear in $Y$, its highest derivative.

DEFINITION 6.1. [M, $\mathcal{V}]$ In the remainder of the paper, fix a finite set $\mathcal{M}$ of $\prec-m o n i c$ functions of which are $\mathbb{F}$ analytic functions on some subset $\mathcal{V}$ of $J^{r}\left(\mathbb{F}^{n}, \mathbb{F}^{m}\right)$ for some finite $r$. The subset is connected and open in the usual $\mathbb{F}$ Euclidean topology.

Definition 6.2. [Principal and Parametric Derivatives] The principal derivatives of $\mathcal{M}$ are defined as

$\operatorname{Prin\mathcal {M}}:=\left\{v \in \Omega \mid \exists f \in \mathcal{M}\right.$ and $\alpha \in \mathbb{N}^{n}$ with $\left.v=\operatorname{HD}^{\alpha} f\right\}$

The parametric derivatives of $\mathcal{M}$, which we denote Par $\mathcal{M}$, are those derivatives that are not principal.

The parametric and principal derivatives enable us to specify initial data, that will be important in the existence and uniqueness theorem.

Definition 6.3. A specification of initial data for $\mathcal{M}$ is a map

$$
\phi:\{x\} \cup \operatorname{Par} \mathcal{M} \rightarrow \mathbb{F}
$$

For $x^{0} \in \mathbb{F}^{m}$, we say that $\phi$ is a specification at $x^{0}$ if

$$
\phi(x):=\left(\phi\left(x_{1}\right), \phi\left(x_{2}\right), \cdots, \phi\left(x_{m}\right)\right)=x^{0} .
$$

For an analytic function $g$ on jet space, let $\phi(g)$ be the function of the principal derivatives obtained from $g$ by evaluating $x$ and the parametric derivatives using $\phi$ :

$$
\phi(g):=g\left(\phi(x),(\phi(v))_{v \in \operatorname{Par} \mathcal{M}) .}\right.
$$

Definition 6.4. [Riquier Basis] $\mathcal{M}$ is called a Riquier Basis if for all $\alpha, \alpha^{\prime} \in \mathbb{N}^{m}$ and $f, f^{\prime} \in \mathcal{M}$ with $\operatorname{HD} D^{\alpha} f=$ $\mathrm{HD} \boldsymbol{D}^{\alpha^{\prime}} f^{\prime}$, the integrability condition $\boldsymbol{D}^{\alpha} f-\boldsymbol{D}^{\alpha^{\prime}} f^{\prime}$ is reduced to zero by a sequence of one-step reductions by members of $\mathcal{M}$.

See [19] for the definition of one-step reduction used above. Recall that $\mathcal{M}$ and $\mathcal{V}$ are as given in Definition 6.1.
Theorem 6.5 (Formal Riquier Existence Theorem). Let $\mathcal{M}$ be a Riquier Basis such that each $f \in \mathcal{M}$ is polynomial in the principal derivatives. For $x^{0} \in \mathbb{F}^{n}$, let $\phi$ be a specification of initial data for $\mathcal{M}$ at $x^{0}$ such that $\phi(f)$ is well-defined for all $f \in \mathcal{M}$. Then there is formal power series solution $u(x) \in \mathbb{F}\left[\left[x-x^{0}\right]\right]^{n}$ to $\mathcal{M}$ at $x^{0}$ such that $\boldsymbol{D}^{\alpha} u^{i}\left(x^{0}\right)=\phi\left(v_{\alpha}^{i}\right)$ for all $v_{\alpha}^{i} \in \operatorname{Par} \mathcal{M}$. Furthermore, every formal power series solution to $\mathcal{M}$ at $x^{0}$ may be obtained in this way for some $\phi$.

Note that the set of integrability conditions given by Definition 6.4 is generally infinite. This infinite number of conditions is shown in [18] to be a consequence of a finite set of integrability conditions given below; thus enabling finite implementation [27]. Further more refined redundancy criteria for integrability conditions are given in [27].

Definition 6.6. Let $f, f^{\prime} \in \mathcal{M}$ with $\operatorname{HD} f=D^{\alpha} u^{i}$ and $\mathrm{HD} f^{\prime}=D^{\alpha^{\prime}} u^{i^{\prime}}$, and $\beta$ be the least common multiple of $\alpha$ and $\alpha^{\prime}$. Then if $i=i^{\prime}$, define the minimal integrability condition of $f$ and $f^{\prime}$ to be $\operatorname{IC}\left(f, f^{\prime}\right)=\boldsymbol{D}^{\beta-\alpha} f-\boldsymbol{D}^{\beta-\alpha^{\prime}} f^{\prime}$. If $i \neq i^{\prime}$, then $\operatorname{IC}\left(f, f^{\prime}\right)$ is said to be undefined.

See [19] for the definition of reduction used below.

THEOREM 6.7. Suppose that for each pair $f, f^{\prime} \in \mathcal{M}$ with $\operatorname{IC}\left(f, f^{\prime}\right)$ well-defined we have $\operatorname{IC}\left(f, f^{\prime}\right)$ is reduced to 0 by a sequence of one-step reductions. Then $\mathcal{M}$ is a Riquier Basis.

\subsection{Implicit Riquier Existence Theorem}

We know that for ODE if the Jacobian matrix is nonsingular, Pryce's method can successfully construct the unique local solution at a given consistent initial point. Now let us consider the PDE case. We show that if $\mathcal{J}$ is non-singular at some point $p$, which satisfies system $\mathbf{D}_{t}^{c} R$, then any order derivative of each $u^{j}$ is determined by $p$. So the Taylor series coefficients of the solution passing through $p$ can be computed to arbitrary order.

For each dependent variable we have a ranking of type (9). To apply the Riquier Existence Theorem, we need to merge these partial rankings (9) to a total ranking which is consistent with all the partial rankings.

Proposition 6.8. Let the leading class derivatives of $R$ be $\left\{\theta_{1}, \ldots, \theta_{m}\right\}$ and let $B$ be the set of all the other derivatives of $R$. Then there exists a positive ranking $\prec$ which satisfies the partial ranking (9) and $\theta_{1} \succ \theta_{2} \succ \cdots \succ \theta_{m}$ and each $\theta_{i}$ is greater than any $b \in B$.

Proof. Case 1: $m \geq n$. Suppose the dependent variable index of $\theta_{i}$ is $i$ and $t=x_{1}$. If the dependent and independent variable indices do not satisfy this condition, then it can be satisfied after a permutation of the variables. Let $\left(\begin{array}{c}I^{m \times m} \\ X^{n \times m}\end{array}\right)=\left(\psi\left(\theta_{1}\right), \ldots, \psi\left(\theta_{m}\right)\right)$. And suppose $c$ is the maximum entry of $X$. Then let $M^{\prime}=c \cdot 1^{m \times m}-\left(\begin{array}{c}X \\ 0\end{array}\right)^{m \times m}$. Finally we construct an $(m+1) \times(m+n)$ matrix $M=$ $\left(\begin{array}{cc}M^{\prime} & I^{n \times n} \\ \mathbf{v} & 0\end{array}\right)$, where $\mathbf{v}=(m, m-1, \cdots, 1)$. All the entries of $M$ are non-negative. Suppose $\theta, \tau \in \Omega$ and $\theta \neq \tau$. One case is they have different dependent variables, then at least the last coordinates of $M \cdot \psi(\theta)$ and $M \cdot \psi(\tau)$ are 
different. The other case is that they have the same dependent variable. Then their ranks are determined by the last $n$ columns of $M$, which is the lexical order over independent variables. In this case, $M \cdot \psi(\theta) \neq M \cdot \psi(\tau)$. So $M$ is a matrix representation of a ranking which satisfies Ranking (9).

Suppose $i<j$, we can check $\theta_{i} \succ \theta_{j}$. Since $\left(\begin{array}{c}\gamma_{i} \\ m-i+1\end{array}\right)=$ $M \cdot \psi\left(\theta_{i}\right)>M \cdot \psi\left(\theta_{j}\right)=\left(\begin{array}{c}\gamma_{j} \\ m-j+1\end{array}\right)$, where $\gamma_{j}=M_{j}^{\prime}+$ $\left(\begin{array}{c}X_{j} \\ 0\end{array}\right)=c \cdot 1^{m \times 1}=\gamma_{i}$.

Suppose $\tau \in B$ with dependent variable $u^{i}$, we can show $\theta_{j} \succ \tau$, for any $j$. Since $\prec_{M}$ satisfies Ranking (9),

$\left(\begin{array}{c}\gamma_{\tau} \\ m-i+1\end{array}\right)=M \cdot \psi(\tau)<M \cdot \psi\left(\theta_{i}\right)=\left(\begin{array}{c}\gamma_{i} \\ m-i+1\end{array}\right)$, which implies $\gamma_{\tau}<\gamma_{i}$. So $\gamma_{\tau}<\gamma_{j}=\gamma_{i}$, for any $1 \leq j \leq m$.

Therefore, $M \cdot \psi(\tau)<M \cdot \psi\left(\theta_{j}\right)$, which implies for any $\theta_{j}$ and any $\tau \in B$ we have $\tau \prec_{M} \theta_{j}$.

Case 2: $m<n$. In the proof, we only need to change the construction slightly by setting $M^{\prime}=c \cdot 1^{n \times m}-X$. Similarly we construct an $(n+1) \times(m+n)$ matrix $M=$ $\left(\begin{array}{cc}M^{\prime} & I^{n \times n} \\ \mathbf{v} & 0\end{array}\right)$

Lemma 6.9. Let $C=\left(\begin{array}{c}A^{n \times m} \\ B^{\ell \times m}\end{array}\right)$ and $n+\ell \leq m$. If $C$ is a full rank matrix, then any rank $n$ square sub-matrix of $A$ can be extended to a rank $n+\ell$ square sub-matrix of $C$.

Proof. Because $C$ is a full rank matrix and $n+\ell \leq m$, $\operatorname{rank}(C)=n+\ell$. Suppose the first $n$ columns of $A$ form a full rank matrix, so the first $n$ columns of $C$ are linearly independent. A set of linearly independent columns can be extended to a basis of the column space of $C$. Hence we can find $\ell$ columns which generate a basis for the column space of $C$ together with the first $n$ columns.

LEMMA 6.10. Let $R$ be a square $\mathbb{F}$-analytic system of PDE. Suppose the maximal transversal value of $\left(\sigma_{i j}\right)(R)$ exists. Let $\boldsymbol{D}_{t}^{c} R$ be the system obtained by the t-prolongation method of Section 5. If $\mathcal{J}_{k_{c}}$ is nonsingular at some point $p$ in $Z\left(\boldsymbol{D}_{t}^{c} R\right)$, then there exists a positive ranking $\prec$ that determines a local solved form $w^{(i)}=f^{(i)}(z)$ for each block $B_{i}$, such that $\boldsymbol{D}_{t} w^{(i-1)} \subseteq w^{(i)}$.

Proof. Because $\mathcal{J}_{k_{c}}$ is nonsingular at $p$, each $\mathcal{J}_{i}$ is full rank by Proposition 5.2. So $B_{0}$ is full rank and we can find an invertible sub-matrix $M_{0}$ of $\mathcal{J}_{0}$, and solve for the corresponding leading variables $w^{(0)}$ locally, which are $t$ derivatives of the dependent variables, by using the Implicit Function Theorem. Let the solved form be $w^{(0)}=f^{(0)}(z)$. Let $S_{0}$ be the set of the dependent variables of $w^{(0)}$. For the next block $B_{1}$ we can choose an invertible sub-matrix $M_{1}$ of $\mathcal{J}_{1}$ which contains $M_{0}$ by Lemma 6.9. Let $S_{i}$ is the set of dependent variables of $w^{(i)} \backslash\left(S_{0} \cup \cdots \cup S_{i-1}\right)$.

Continue the process until the last block $B_{k_{c}}$. And we can check the union of all $S_{i}$ is the set of all dependent variables.

Suppose that $U_{k_{c}}=\left\{\theta_{1}, \cdots, \theta_{m}\right\}$ and (after appropriate re-indexing) satisfies the condition: for any $1 \leq i<j \leq m$, if the dependent variables of $\theta_{i}$ and $\theta_{j}$ belong to $S_{p}$ and $S_{q}$ respectively then $p \leq q$. We can define a positive ranking $\prec$ by the Proposition 6.8 such that the solved term is leading variable for each solved form in $\left\{w^{(i)}=f^{(i)}(z)\right\}$.

For background on the Implicit Function Theorem and related results needed on what follows please see [5,9]. Let $w_{0} \in \mathbb{F}^{k}, z_{0} \in \mathbb{F}^{\ell}$ and $\mathcal{U} \subset \mathbb{F}^{k} \times \mathbb{F}^{\ell}$ be a neighborhood of $\left(w_{0}, z_{0}\right)$

Let $F: \mathcal{U} \rightarrow \mathbb{F}^{k}$ be an analytic function with $F\left(w_{0}, z_{0}\right)=$ 0 and $\operatorname{rank} \frac{\partial F}{\partial w}=k$ at $\left(w_{0}, z_{0}\right) \in \mathcal{U}$. That is, the Jacobian of $F$ has maximal rank with respect to $w$ at $\left(w_{0}, z_{0}\right)$. Then by the Implicit Function Theorem there exists an analytic function $f: \mathbb{F}^{\ell} \rightarrow \mathbb{F}^{k}$, such that the zero set of $\{(w, z): F(w, z)=0\}$ is equivalent to $\{(w, z): w=f(z)\}$ in a neighborhood of $\mathcal{N}$ of $\left(w_{0}, z_{0}\right)$.

We have the following simple consequence.

REMARK 6.11. There exists a neighborhood of $\mathcal{N}$ of $\left(w_{0}, z_{0}\right)$ and an analytic function $H: \mathcal{N} \rightarrow \mathbb{F}^{k \times k}$ such that

$$
F(w, z)=H(w, z)(w-f(z))
$$

and $H(w, z)$ is invertible in $\mathcal{N}$.

THEOREM 6.12. Let $R$ be a square $\mathbb{F}$-analytic system of PDE. Suppose the maximal transversal value of $\left(\sigma_{i j}\right)(R)$ exists. Let $\boldsymbol{D}_{t}^{c} R$ be the system computed by t-prolongation method. If $\mathcal{J}_{k_{c}}$ is nonsingular at some point $p$ in $Z\left(\boldsymbol{D}_{t}^{c} R\right)$, then $\boldsymbol{D}_{t}^{c} R$ is an Implicit Riquier Basis.

Proof. By Proposition 6.8, there is a ranking in which all leading derivatives are pure $t$-derivatives. And by Lemma 6.10 , there exists a solved form $w=f(z)$ of $\mathbf{D}_{t}^{c} R$ in a sufficiently small neighborhood $\mathcal{N}_{p}$, where $w$ is the union of all $w^{(i)}$ defined in Lemma 6.10. We will show that $w=f(z)$ is a Riquier Basis in $\mathcal{N}_{p}$. First note that the principal derivatives of $w=f(z)$ are given by $w$. Thus $w=f(z)$ is certainly polynomial in $w$ as required by Theorem 6.5. Secondly, it remains to prove that the integrability conditions of $w=f(z)$ are satisfied. So without loss of generality, we consider two particular equations $\hat{w}-\hat{f}(z)=0$ and $\tilde{w}-\tilde{f}(z)=0$ with $\left(\mathbf{D}_{t}\right)^{\gamma} \hat{w}=\tilde{w}$. By Theorem 6.7, the corresponding integrability condition is $\left(\mathbf{D}_{t}\right)^{\gamma}(\hat{w}-\hat{f}(z))-(\tilde{w}-\tilde{f}(z))$. By the more refined redundancy criterion given in Corollary 5.3.2 of [18], this can be reduced to case $\gamma=1$ :

$$
\mathbf{D}_{t}(\hat{w}-\hat{f}(z))-(\tilde{w}-\tilde{f}(z))
$$

where $\hat{w}-\hat{f}(z)=0$ and $\tilde{w}-\tilde{f}(z)=0$ are two particular equations out of the solved forms $w^{(i-1)}=f^{(i-1)}(z)$ and $w^{(i)}=f^{(i)}(z)$ respectively, with $\mathbf{D}_{t} \hat{w}=\tilde{w}$.

Remark 6.11 implies that $w^{(i)}-f^{(i)}(z)=H_{i}^{-1} \cdot B_{i}$ in $\mathcal{N}_{p}$. Thus $\tilde{w}-\tilde{f}(z)=\tilde{h} \cdot B_{i}$ in $\mathcal{N}_{p}$, for some analytic function vector $\tilde{h}$. Similarly $\hat{w}-\hat{f}(z)=\hat{h} \cdot B_{i-1}$ in $\mathcal{N}_{p}$, for some analytic function vector $\hat{h}$. Then (18) is

$$
\mathbf{D}_{t}\left(\hat{h} \cdot B_{i-1}\right)-\tilde{h} \cdot B_{i}
$$

which has the general form

$$
\mathbf{D}_{t} \hat{h} \cdot B_{i-1}+\hat{h} \cdot \mathbf{D}_{t} B_{i-1}-\tilde{h} \cdot B_{i}
$$

Because $\mathbf{D}_{t} B_{i-1} \subseteq B_{i},(18)$ is zero on $\mathcal{N}_{p} \cap Z\left(\mathbf{D}_{t}^{c} R\right)$, which is equivalent to $\{(w, z): w=f(z)\} \cap \mathcal{N}_{p}$. So (18) is zero when $w=f(z)$ in $\mathcal{N}_{p}$, which means (18) can be reduced to zero by $w=f(z)$ (due to properties of analytic functions). Therefore $\mathbf{D}_{t}^{c} R$ is an implicit Riquier Basis in $\mathcal{N}_{p}$. 
REMARK 6.13. Suppose the maximal transversal value of a signature matrix exists. Then the prolongation step vector $c$ is determined only by the signature matrix rather than the algebraic degree and coefficients. So a signature matrix corresponds to a class of t-dominated PDE. For a square polynomially nonlinear PDE system $R$ in such a class, if the coefficient of each term is generic, then at a generic point in the variety defined by $\boldsymbol{D}_{t}^{c} R$ in Jet space, the Jacobian matrix $\mathcal{J}_{k_{c}}$ is non-singular. This means the t-prolongation method can be applied to a large class of PDE together with Proposition 4.2.

\section{APPROXIMATING POINTS ON ZERO SETS OF PDE}

The method we have developed depends on finding a point $p$ on the zero set $Z(R)$ of the PDE system $R$ to test that the relevant Jacobian's are non-singular. Their non-singularity at a point (and thus in a neighbourhood) ensures that the conditions for local existence and uniqueness are satisfied for Theorem 6.12.

We consider polynomially nonlinear PDE as polynomial systems in Jet space. Our tool to numerically solve polynomial systems is homotopy continuation. In [24], a new field "Numerical Algebraic Geometry" was described which led to the development of homotopies to describe all irreducible components (all meaning: for all dimensions) of the solution set of a polynomial system by witness sets. These methods have been implemented in PHCpack [25].

Following Pryce's idea in [13], we can compute $p \in Z(R)$ by exploiting the triangular block structure of the PDE system after the partial prolongation (see Table 1).

REMARK 7.1. In the case of ODE, we can compute the witness points of $B_{0}$, which is the projection of the variety to the subspace, then substitute the solutions into $B_{1}$ to extend the solutions to higher dimensional space. Continuing this process, we can find the the witness points of non-singular components. This way is more efficient than solving the whole polynomial system directly. Let $R$ be a polynomially ODE $\left\{R_{1}, \ldots, R_{m}\right\}$ with total degree $d$. Then the Bezout bound of $D_{t}^{c}(R)$ in Jet space is $d^{C} d^{m}$, where $C=\sum c_{i}$. However if we solve it by bottom up substitution it only has at most $d^{m}$ homotopy continuation paths to track, since any nonlinear equation will be linear with respect to highest Jet variables after prolongation.

Usually applications involve finding real solutions. For real differential polynomial systems using our approach, we need to find points on a real variety. Real algebraic geometry is a rapidly developing area with many recent developments detailed in the book [3]. There are several techniques for compact varieties while approaches are less well-developed in the non-compact case. Lu [12] uses homotopy continuation in $\mathbb{C}$ to decompose varieties first over $\mathbb{C}$, then obtains points on the real curves embedded in the 1-dimensional complex components. In our experiments, we heuristically selected some proper real linear equations to slice the variety to obtain real points on the zero set of the PDE.

\section{EXAMPLES}

The $t$-prolongation procedure for ODE and PDE was implemented in Maple10. The integer linear programming involved using Maple10's LPSolve command. As a feasibility

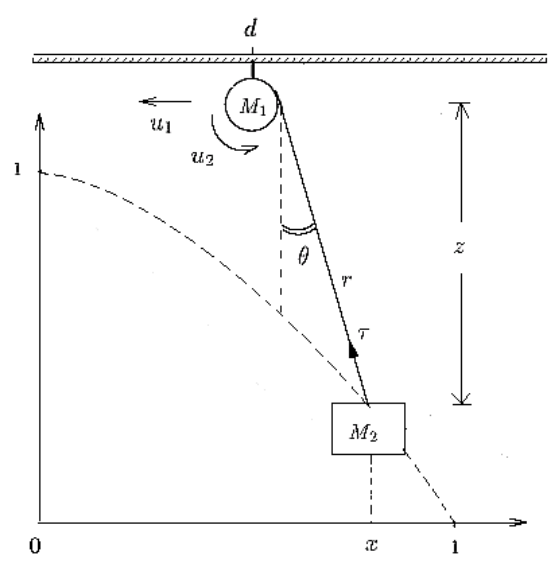

Figure 2: Control of a Crane

test we applied the code to a Test Set of Visconti [26] containing 27 DAE representing diverse applications, with index ranging from 1 to 6 . The procedure successfully identified index consistent with Visconti's results for 21 of the DAE. The LP problems were solved in less, and often much less, than one second. Our 6 failures were due to: 3 non-square system; 3 systems with singular Jacobians. Like other standard DAE approaches, Visconti required the user to supply an initial guess for a consistent initial point, and then GaussNewton iteration was applied. An example is given below.

EXAMPLE 8.1. [ODE for a Crane] This model which is illustrated in Figure 8.1, is discussed in [4]. The problem is to determine the horizontal velocity $u_{1}(t)$ of a winch of mass $M_{1}$, and the angular velocity $u_{2}(t)$ of the winch so that the attached load $M_{2}$ moves along a prescribed path.

The equations of motion are given by [4] and also by Visconti [26] with unknowns $\left\{x, x^{\prime}, z, z^{\prime}, d, d^{\prime}, r, r^{\prime}, \theta, \tau, u_{1}, u_{2}\right\}$ :

$$
\begin{aligned}
x_{t}-x^{\prime}=0, \quad z_{t}-z^{\prime}=0, \quad d_{t}-d^{\prime}=0, \quad r_{t}-r^{\prime} & =0 \\
M_{2} x_{t}^{\prime}+\tau \sin (\theta)=0, \quad M_{1} d_{t}^{\prime}+C_{1} d_{t}-u_{1}-\tau \sin (\theta) & =0 \\
M_{2} z_{t}^{\prime}+\tau \cos (\theta)-m g=0, \quad J r_{t}^{\prime}+C_{2} r_{t}+C_{3} u_{2}-C_{3}{ }^{2} \tau & =0 \\
r \sin (\theta)+d-x=0, \quad r \cos (\theta)-z & =0 \\
H_{1}(x, z, t)=0, \quad H_{2}(x, z, t) & =0 .
\end{aligned}
$$

The prescribed path of the mass $M_{2}$ is described by an algebraic equations $\left\{H_{1}=0, H_{2}=0\right\}$. The winch has moment of inertia $J$ and is attached with a cable of length $r(t)$, making an angle $\theta(t)$ to the vertical.

Substitute $\sin (\theta)$ and $\cos (\theta)$ by $s(t)$ and $c(t)$ respectively to convert the ODE to an algebraic differential system, and introduce an extra equation $s(t)^{2}+c(t)^{2}=1$. Applying the $t$-prolongation method and our Maple program, we obtain

$$
\begin{aligned}
& d_{1}=4, d_{2}=3, d_{3}=4, d_{4}=3, d_{5}=2, d_{6}=1, d_{7}=2, \\
& d_{8}=1, d_{9}=2, d_{10}=0, d_{11}=0, d_{12}=2, d_{13}=2 ; \\
& c_{1}=3, c_{2}=3, c_{3}=1, c_{4}=1, c_{5}=2, c_{6}=2, c_{7}=0, \\
& c_{8}=0, c_{9}=2, c_{10}=2, c_{11}=4, c_{12}=4, c_{13}=2 .
\end{aligned}
$$

Since $d_{10}$ and $d_{11}$ are equal to zero, we need to prolong one more time to reduce the system to ODE. For this example we have index 5 in agreement with Visconti. Note that the result does not depend on the coefficients and degrees of $H_{1}, H_{2}$ 
since the signature matrix only requires the differential orders of $H_{1}, H_{2}$ which are both 0 .

To simply illustrate how to use the output, we choose a path $\left\{H_{1}=0, H_{2}=0\right\}$ of the mass $M_{2}$ which is described by a parameterized system $x(t)=1-t^{2}, z(t)=1-t$. After the partial prolongation we obtain 13 ODE and 39 algebraic constraints. The total Bezout degree of the constraints is 65536, however it has block triangular structure which enable us to solve it by bottom up substitution.

Let the initial time $t$ be .396 , we obtain 4 witness points using PHCpack. We choose one as the initial point which is $x(.396)=.843, z(.396)=.604, d(.396)=.601, s(.396)=$ $.371, c(.396)=.928, r(.396)=0.650$. Note that if the degree of $H_{1}, H_{2}$ is $d$, there are at most $4 d^{2}$ witness points during the computation by Remark 7.1. The computational difficulty of this problem for the symbolic differential elimination algorithm Rifsimp explosively increases with the degree $d$ of $H_{1}, H_{2}$ in comparison with the $t$-prolongation method.

Finally we numerically solve the ODE together with this initial condition using dsolve in Maple10 with its implicit option. The integral curve of $x(t), z(t)$ is very close to the curve $\left(1-t^{2}, 1-t\right)$.

EXAMPLE 8.2. [Pendulum Curtain PDE] Applying the t-prolongation method to Example 5.1 gives:

$$
\begin{aligned}
& c_{1}=0, \quad c_{2}=0, \quad c_{3}=2 ; \\
& d_{1}=2, \quad d_{2}=2, \quad d_{3}=0 .
\end{aligned}
$$

The main point, is that the analysis for this PDE example, is virtually identical to that for the classical pendulum (see Pryce [13] for those details). Essentially the analysis, indicates that the constraint should be differentiated twice to yield an implicit Riquier Basis:

$$
\begin{aligned}
X_{t t}+\lambda X & =\kappa X_{s s} \\
Y_{t t}+\lambda Y+g & =\kappa Y_{s s} \\
X X_{t t}+Y Y_{t t}+X_{t}^{2}+Y_{t}^{2} & =0 \\
X X_{t}+Y Y_{t} & =0 \\
X^{2}+Y^{2}-1 & =0 .
\end{aligned}
$$

The top block $B_{2}$ of the system is the first three equations of (23). The blocks $B_{1}$ and $B_{0}$ are the 4 th and 5 th equations of (23) respectively.

Then the system has Jacobian matrix with respect to $X_{t t}$, $Y_{t t}, \lambda$ which is full rank. This is also obvious by inspection, without using the generalization of Pryce's method. We include it here, so that the reader can see it working on an example, which is closely related a one of the fundamental examples of DAE theory. We note that a change of coordinates to cylindrial coordinates $X=\sin (\theta(s, t)), Y=-\cos (\theta(s, t))$, considerably simplifies the problem. However, in general, such coordinate changes cannot be algorithmically made to eliminate all constraints for PDE.

In summary we obtain an explicit hyperbolic system on a system of constraints. Just as an explicit ODE is uncovered in the analysis of the classical pendulum, an explicit Hyperbolic System of PDE is obtained in the Pendulum Curtain example. We solved this system using Wittkopf's finite difference code in Maple10. We performed experiments with various initial and boundary conditions and values of $\kappa$. One of these was for an exponential bump located in the middle of the s-range, where the curtain is released from rest. As expected this forms two waves, moving in opposite directions.
If the coefficient $\kappa$ of the $X_{s s}$ and $Y_{\text {ss }}$ terms are set close to zero (i.e. $\kappa \approx 0$ ) then as expected the pendulum motion rather than the wave motion dominates.

EXAMPLE 8.3. [Changing the Coordinates] The equation below is both $x$ and $y$ dominated. However for small $\epsilon_{1}$, $\epsilon_{2}$, the resulting Jacobians in our method are poorly conditioned.

$$
\epsilon_{1} u_{x x}+u_{x y}+\epsilon_{2} u_{y y}=0
$$

The problem is well conditioned after a coordinate change (see Proposition 4.2).

\section{DISCUSSION}

A significant problem in the development of symbolicnumeric differential elimination methods is to create methods to control the growth of prolongations. Although much progress has been made on the symbolic case [1], little has been done for symbolic-numeric case.

In the current work we define a class of systems, for which only prolongations with respect to a single independent variable $t$ are needed.

We generalized Pryce's technique in the framework of Riquier Bases. Riquier's classical approach has fallen out of favor in recent times, since for a purely symbolic implementation, it is limited to systems linear in their highest derivatives and modern symbolic alternatives now exist $[2$, 27]. However in our article, Riquier's approach makes a comeback, by using the Implicit Function Theorem, which requires points on the zero set of the system. For systems of differential polynomials over $\mathbb{C}$, we can use homotopy methods from Numerical Algebraic Geometry to compute approximations to such points [24]. For systems of differential polynomials over $\mathbb{R}$, there are also rapidly evolving methods $[12,3]$. For analytic systems, methods are less systematic but progress can be made using Gaussian-Newton iteration from initial guesses close enough to a solution.

It may seem strange that such implicit representations could be useful, especially since the representations given by such symbolic elimination methods as [2] provide output systems in much closer to explicit solved or triangular form. However such eliminations can often cause severe expression swell. The Pryce method, appears to find a balance between working implicitly, while at the same time uncovering and exploiting the block structure of a system. Finally we note that such implicit representations, are usually the choice in the numerics community. Solving a constant matrix system, at the intermediate steps of a numerical integration, is often preferred over first symbolically inverting, then evaluating the explicit solution at those intermediate steps.

The disadvantages of our method, include its limitation to square and $t$-dominated systems. It also has the disadvantage, that its a local method, and not a universal method and does not pursue all singular cases as is possible using $[2,7]$. For example when the method is applied to $\left(u_{t}\right)^{2}+t u_{t}-u=0$ it locates a generic initial point, and does not identify the fact that this equation has a singular solution. In addition a linear combination of the input system will destroy the sparse structure of the signature matrix. However this can be detected by a rank test and the hidden equations can be constructed by the methods we give in [28].

The implicit Riquier Bases obtained by our method are a type of formally integrable system. Such bases only give 
local, and sometimes, unnatural boundary and initial conditions. We direct the reader to Krupchyk et al. [10, 11] for very interesting work on linking formal properties (such as formal integrability and involutivity) to elliptic BVP.

Our method provides a bridge between ODE techniques and PDE techniques. In this paper we generalized a method of Pryce and Pantiledes, to PDE. An obvious future work, is to attempt the same with other ODE methods. We are investigating PDE models arising as more realistic cases of DAE system, for which our $t$-prolongation method promises to be practically useful.

\section{Acknowledgement}

The authors gratefully acknowledge support for this work from the NSF funded IMA Thematic Year on Applications of Algebraic Geometry, and also acknowledge support from Reid's NSERC grant. We especially thank Jan Verschelde and John McPhee for many discussions and valuable assistance. We also thank Silvana Ilie, Eric Schost and Allan Wittkopf for helpful discussions. We thank the Referees for many helpful comments.

\section{REFERENCES}

[1] F. Boulier. Réécriture algébrique dans les systèmes d'équations différentielles en vue d'applications dans les Sciences du Vivant. Habilitation Thesis, 2006.

[2] F. Boulier, D. Lazard, F. Ollivier, and M. Petitot. Representation for the radical of a finitely generated differential ideal. Proc. ISSAC 1995. ACM Press. 158-166, 1995.

[3] S. Basu, R. Pollack and M. Roy. Algorithms in Real Algebraic Geometry, Springer, 2003.

[4] S.L. Campbell. High index differential algebraic equations. J. Mech. Struct. and Machines, 23: pp 199-222, 1995.

[5] R.C. Gunning and H. Rossi. Analytic functions of several complex variables. Prentice-Hall, 1965.

[6] S. Ilie, R.M. Corless and G. Reid. Numerical solutions of index-1 differential algebraic equations can be computed in polynomial time. Numerical Algorithms, Vol. 41 (2), pp. 161-171, 2006.

[7] E. Hubert. Notes on triangular sets and triangulation-decomposition algorithms II: Differential Systems. Symbolic and Numerical Scientific Computations, Edited by U. Langer and F. Winkler. LNCS, volume 2630, Springer-Verlag Heidelberg, 2003.

[8] E. Kolchin. Differential Algebra and Algebraic Groups. Academic Press, New York, 1973.

[9] S. Krantz and H. Parks. A Primer of Real Analytic Functions. Basler Lehrbücher, 2002.

[10] K. Krupchyk and J. Tuomela. Shapiro-Lopatinskij Condition for Elliptic Boundary Value Problems. LMS J. Comput. Math. 9 (2006) pp. 287-329.

[11] K. Krupchyk, W. Seiler and J. Tuomela. Overdetermined Elliptic PDEs. Found. Comp. Math. 6 (2006), No. 3, pp 309-351.

[12] Y. Lu. Finding all real solutions of polynomial systems. Ph.D. Thesis, University of Notre Dame, 2006 (Submitted).
[13] J.D. Pryce. A Simple Structure Analysis Method for DAEs. BIT, vol 41, No. 2, pp. 364-394, 2001.

[14] E. Mansfield. Differential Gröbner Bases. Ph.D. thesis, Univ. of Sydney, 1991.

[15] G. Reid, C. Smith, and J. Verschelde. Geometric completion of differential systems using numeric-symbolic continuation. SIGSAM Bulletin 36(2):1-17, 2002.

[16] G. Reid, J. Verschelde, A.D. Wittkopf and W. Wu. Symbolic-Numeric Completion of Differential Systems by Homotopy Continuation. Proc. ISSAC 2005. ACM Press. 269-276, 2005.

[17] G.J. Reid, P. Lin, and A.D. Wittkopf. Differential elimination-completion algorithms for DAE and PDAE. Studies in Applied Math. 106(1): 1-45, 2001.

[18] C.J. Rust, Rankings of derivatives for elimination algorithms and formal solvability of analytic partial differential equations, Ph.D. Thesis, University of Chicago, 1998.

[19] C.J. Rust, G.J. Reid, and A.D. Wittkopf. Existence and uniqueness theorems for formal power series solutions of analytic differential systems. Proc. ISSAC 99. ACM Press. 105-112, 1999.

[20] F. Schwarz. Janet bases for symmetry groups, in: Groebner bases and applications. London Math. Soc., LNS 251, Cambridge Univ. Press, 221-234, 1998.

[21] A. Sedoglavic. A probabilistic algorithm to test local algebraic observability in polynomial time. $J$. Symbolic Computation 33(5): 735-755, 2002.

[22] W.M. Seiler. Involution - The formal theory of differential equations and its applications in computer algebra and numerical analysis. Habilitation Thesis, Univ. of Mannheim, 2002.

[23] Pengfei Shi, John McPhee. Symbolic Programming of a Graph-Theoretic Approach to Flexible Multibody Dynamics. Mechanics of Structures and Machines, 30(1), 123-154, 2002.

[24] A.J. Sommese and C.W. Wampler. The Numerical solution of systems of polynomials arising in engineering and science. World Scientific Press, Singapore, 2005

[25] J. Verschelde. Algorithm 795: PHCpack: A general-purpose solver for polynomial systems by homotopy continuation. ACM Transactions on Mathematical Software 25(2): 251-276, 1999.

[26] J. Visconti. Numerical Solution of Differential Algebraic Equations, Global Error Estimation and Symbolic Index Reduction. Ph.D. Thesis. Laboratoire de Modélisation et Calcul. Grenoble. 1999.

[27] A. Wittkopf. Algorithms and Implementations for Differential Elimination. Ph.D. Thesis, Simon Fraser University, 2004.

[28] Wenyuan Wu and Greg Reid. Application of Numerical Algebraic Geometry and Numerical Linear Algebra to PDE. Proc. of ISSAC'06, pages 345-352, ACM 2006. 